\title{
Developing a systems-based framework of the factors influencing dietary and physical activity behaviours in ethnic minority populations living in Europe - a DEDIPAC study
}

Michelle Holdsworth $^{1 *}$ D, Mary Nicolaou ${ }^{2}$, Lars Jørun Langøien ${ }^{3}$, Hibbah Araba Osei-Kwasi ${ }^{1}$, Sebastien F. M. Chastin ${ }^{4}$, F. Marijn Stok ${ }^{5}$, Laura Capranica ${ }^{6}$, Nanna Lien${ }^{7}$, Laura Terragni ${ }^{8}$, Pablo Monsivais ${ }^{9,10}$, Mario Mazzocchi ${ }^{11}$, Lea Maes ${ }^{12}$, Gun Roos ${ }^{13}$, Caroline Mejean ${ }^{14}$, Katie Powell ${ }^{1}$ and Karien Stronks ${ }^{2}$

\begin{abstract}
Background: Some ethnic minority populations have a higher risk of non-communicable diseases than the majority European population. Diet and physical activity behaviours contribute to this risk, shaped by a system of inter-related factors. This study mapped a systems-based framework of the factors influencing dietary and physical activity behaviours in ethnic minority populations living in Europe, to inform research prioritisation and intervention development.

Methods: A concept mapping approach guided by systems thinking was used: i. Preparation (protocol and terminology); ii. Generating a list of factors influencing dietary and physical activity behaviours in ethnic minority populations living in Europe from evidence (systematic mapping reviews) and 'eminence' (89 participants from 24 academic disciplines via brainstorming, an international symposium and expert review) and; iii. Seeking consensus on structuring, rating and clustering factors, based on how they relate to each other; and iv. Interpreting/utilising the framework for research and interventions. Similar steps were undertaken for frameworks developed for the majority European population.

Results: Seven distinct clusters emerged for dietary behaviour (containing 85 factors) and 8 for physical activity behaviours (containing 183 factors). Four clusters were similar across behaviours: Social and cultural environment; Social and material resources; Psychosocial; and Migration context. Similar clusters of factors emerged in the frameworks for diet and physical activity behaviours of the majority European population, except for 'migration context'. The importance of factors across all clusters was acknowledged, but their relative importance differed for ethnic minority populations compared with the majority population.

(Continued on next page)
\end{abstract}

\footnotetext{
* Correspondence: michelle.holdsworth@sheffield.ac.uk

${ }^{1}$ Public Health Section, School of Health and Related Research-ScHARR, The

University of Sheffield, Sheffield, UK

Full list of author information is available at the end of the article
} 
(Continued from previous page)

Conclusions: This systems-based framework integrates evidence from both expert opinion and published literature, to map the factors influencing dietary and physical activity behaviours in ethnic minority groups. Our findings illustrate that innovative research and complex interventions need to be developed that are sensitive to the needs of ethnic minority populations. A systems approach that encompasses the complexity of the inter-related factors that drive behaviours may inform a more holistic public health paradigm to more effectively reach ethnic minorities living in Europe, as well as the majority host population.

Keywords: Minority populations, Europe, Migrants, Immigrants, Physical activity, Sedentary behaviour, Diet, Framework, Systems

\section{Background}

Some ethnic minority groups living in Europe have a high prevalence of preventable non-communicable diseases (NCDs), such as obesity, type 2 diabetes and cardiovascular diseases [1-4]. Diet and physical activity behaviours are likely to play a role in their aetiology and differences in these behaviours compared with host populations are well documented [4-10]. However, there are fewer studies of the differences in the underlying factors influencing these behaviours [11-13]. Most studies have focused either on a small number of minority groups or are limited to specific European countries, which makes it challenging to generalise about the nature of any differences [14-20]. It could be argued that the variation between ethnic groups is as great as that between the general European population. Nonetheless, it could be expected that there are commonalities in people's lives who are either first or second generation migrants, which are distinct from the general population [11-13]. Thus, understanding the factors underlying diet and physical activity behaviours in ethnic minority groups is a first step to informing the development of public health interventions that are successful in reaching minority ethnic populations in Europe.

Existing frameworks are insufficient for prioritising research or interventions development, as they either focus on migration and dietary acculturation processes [13] or on a specific health outcome, such as obesity in the whole population [21, 22]. Alternatively, they are based on evidence of ethnic minority populations living outside of Europe, for example, of African descent in the US [21] or Iranians living in Australia [23]. This may offer useful insights, even though contextual differences limit their transferability to Europe. Likewise, focusing only on obesity has the potential to ignore important drivers of the complex system of factors influencing dietary and physical activity behaviours.

A system-based approach has the potential to cast a holistic analytic lens [24] to developing interventions, because it is based on the interrelationship of clusters within a dynamic system. Systems thinking can simply be defined as looking at things in terms of the bigger picture' [25]. Dietary and physical activity behaviours therefore emerge as a property, which cannot be resolved from simple, uni-faceted interventions [26]. Shifts are likely to be required within multiple clusters of factors, even though some of these may only have small effects on individuals, they have the potential to stimulate population changes when combined [26]. Therefore, the aim of the current study was to develop a systems-based framework of the factors influencing dietary and physical activity behaviours in ethnic minority populations living in Europe, to be able to inform research prioritisation and the development of interventions to reach these groups.

\section{Methods}

The framework was constructed as part of the DEDIPACKH (DEterminants of Dlet and Physical ACtivity Knowledge $\mathrm{Hub}$ ) [27] for European populations. Within the DEDIPAC-KH, an inter-disciplinary group focused on the determinants of dietary and physical activity/sedentary behaviours. The task was undertaken at several steps involving scholars with varying academic backgrounds and from different countries (Table 1 for more details).

A total of 89 participants contributed to at least one step of the creation of the framework. This comprised a team focusing on the factors influencing the behaviours of ethnic minority populations ('DEDIPAC ethnic minority team'). Other teams in the DEDIPAC-KH focussed on the factors influencing dietary [28] and physical activity/sedentary behaviours $[29,30]$ of the general European population ('DEDIPAC general population team'). This approach followed earlier successful examples of multidisciplinary partnerships that comprehensively described the factors influencing obesity-related behaviours [31].

The method was guided by concept mapping; drawing on both quantitative [32], and qualitative [33] approaches. Traditionally, quantitative concept maps have been used in health research, yet the case for flexible use of concepts maps has been advocated for, requiring a less rigid and more qualitative approach [33, 34]. Concept mapping is influenced by systems thinking and involves gathering and analysing different types of data 
Table 1 Characteristics of participants in the different concept mapping stages to develop the framework

\begin{tabular}{|c|c|c|c|}
\hline & $\begin{array}{l}\text { Stage i, ii: Preparation and } \\
\text { generation of factors }\end{array}$ & $\begin{array}{l}\text { Stage iii: Structuring, rating and } \\
\text { producing cluster map }\end{array}$ & Stage iv: Interpretation and utilisation \\
\hline Participant numbers & $\begin{array}{l}n=12 \text { ethnic minorities DEDIPAC team } \\
n=21 \text { DEDIPAC general population team }\end{array}$ & $\begin{array}{l}n=12 \text { ethnic minorities DEDIPAC team } \\
n=21 \text { DEDIPAC general population team }\end{array}$ & $\begin{array}{l}\text { International symposium: } n=44 \\
\text { delegates and } n=2 \text { invited experts } \\
\text { Finalisation } n=18 \text { members of } \\
\text { DEDIPAC ethnic minorities and } \\
\text { general population teams }\end{array}$ \\
\hline Fields of expertise & $\begin{array}{l}\text { Agricultural economics } \\
\text { Behavioural economics } \\
\text { Behavioural nutrition } \\
\text { Consumer science } \\
\text { Dietary inequalities } \\
\text { Dietetics } \\
\text { Epidemiology } \\
\text { Exercise physiology } \\
\text { Health inequalities } \\
\text { Medicine } \\
\text { Migrant health } \\
\text { Nutritional epidemiology } \\
\text { Physical activity } \\
\text { Psychology (health, cognitive and social) } \\
\text { Public health nutrition } \\
\text { Social anthropology } \\
\text { Social demography } \\
\text { Social inequalities } \\
\text { Sociology of health }\end{array}$ & $\begin{array}{l}\text { Agricultural economics } \\
\text { Behavioural economics } \\
\text { Behavioural nutrition } \\
\text { Consumer science } \\
\text { Dietary inequalities } \\
\text { Dietetics } \\
\text { Epidemiology } \\
\text { Exercise physiology } \\
\text { Health inequalities } \\
\text { Medicine } \\
\text { Migrant health } \\
\text { Nutritional epidemiology } \\
\text { Physical activity } \\
\text { Psychology (health, cognitive and social) } \\
\text { Public health nutrition } \\
\text { Social anthropology } \\
\text { Social demography } \\
\text { Social inequalities } \\
\text { Sociology of health }\end{array}$ & $\begin{array}{l}\text { Agricultural economics } \\
\text { Behavioural economics } \\
\text { Behavioural nutrition } \\
\text { Behavioural sciences } \\
\text { Consumer science } \\
\text { Dietary inequalities } \\
\text { Dietetics } \\
\text { Epidemiology } \\
\text { Exercise physiology } \\
\text { Food engineering } \\
\text { Health inequalities } \\
\text { Medicine } \\
\text { Migrant health } \\
\text { Nutritional epidemiology } \\
\text { Nutrition science } \\
\text { Physical activity } \\
\text { Physical education and physiotherapy } \\
\text { Physical anthropology } \\
\text { Psychology (health, cognitive and social) } \\
\text { Public health nutrition } \\
\text { Social anthropology } \\
\text { Social demography } \\
\text { Social inequalities } \\
\text { Sociology of health }\end{array}$ \\
\hline Countries & $\begin{array}{l}\text { Belgium } \\
\text { France } \\
\text { Germany } \\
\text { Ireland } \\
\text { Italy } \\
\text { Netherlands } \\
\text { Norway } \\
\text { UK }\end{array}$ & $\begin{array}{l}\text { Belgium } \\
\text { France } \\
\text { Germany } \\
\text { Ireland } \\
\text { Italy } \\
\text { Netherlands } \\
\text { Norway } \\
\text { UK }\end{array}$ & $\begin{array}{l}\text { Australia } \\
\text { Belgium } \\
\text { Denmark } \\
\text { France } \\
\text { Germany } \\
\text { Ireland } \\
\text { Italy } \\
\text { Netherlands } \\
\text { Norway } \\
\text { Poland } \\
\text { Spain } \\
\text { Sweden } \\
\text { UK }\end{array}$ \\
\hline
\end{tabular}

and integrating these with prior research/experience [35]. Concept mapping was selected because it can illustrate how people visualize relationships between concepts within a map [34] and it can be used for research prioritisation [30]. The approach was structured around the four main phases proposed in quantitative concept mapping [32], but a more flexible mixed methodology was employed [34]: i. preparation; ii. generation of factors; iii. Structuring and rating factors into clusters; and iv. interpretation and utilisation of the framework.

\section{Preparation (terminology, protocol)}

The scope and purpose of the study protocol was developed (Fig. 1- step 1) by the DEDIPAC ethnic minority team, in consultation with the DEDIPAC general population team. Consensus was reached on the terminology for the different behaviours and for defining factors/correlates/determinants, so that there was a common understanding across the DEDIPAC-KH. Minority ethnic populations were defined as immigrants/populations of immigrant background from low and middle income countries, population groups from the former Eastern Bloc countries who migrate to other parts of Europe and minority indigenous populations in Europe'.

Three criteria were selected to score each factor, for its priority for future research ('research priority') and for how useful it would be in developing interventions ('expected modifiability'; 'potential effect size on behaviour').

It was also decided how consultation outside of the DEDIPAC network could be undertaken, to ensure that a wide range of viewpoints were considered from diverse disciplines to encourage 'buy in' to the resulting framework [32]. The concept mapping process does not stipulate that 


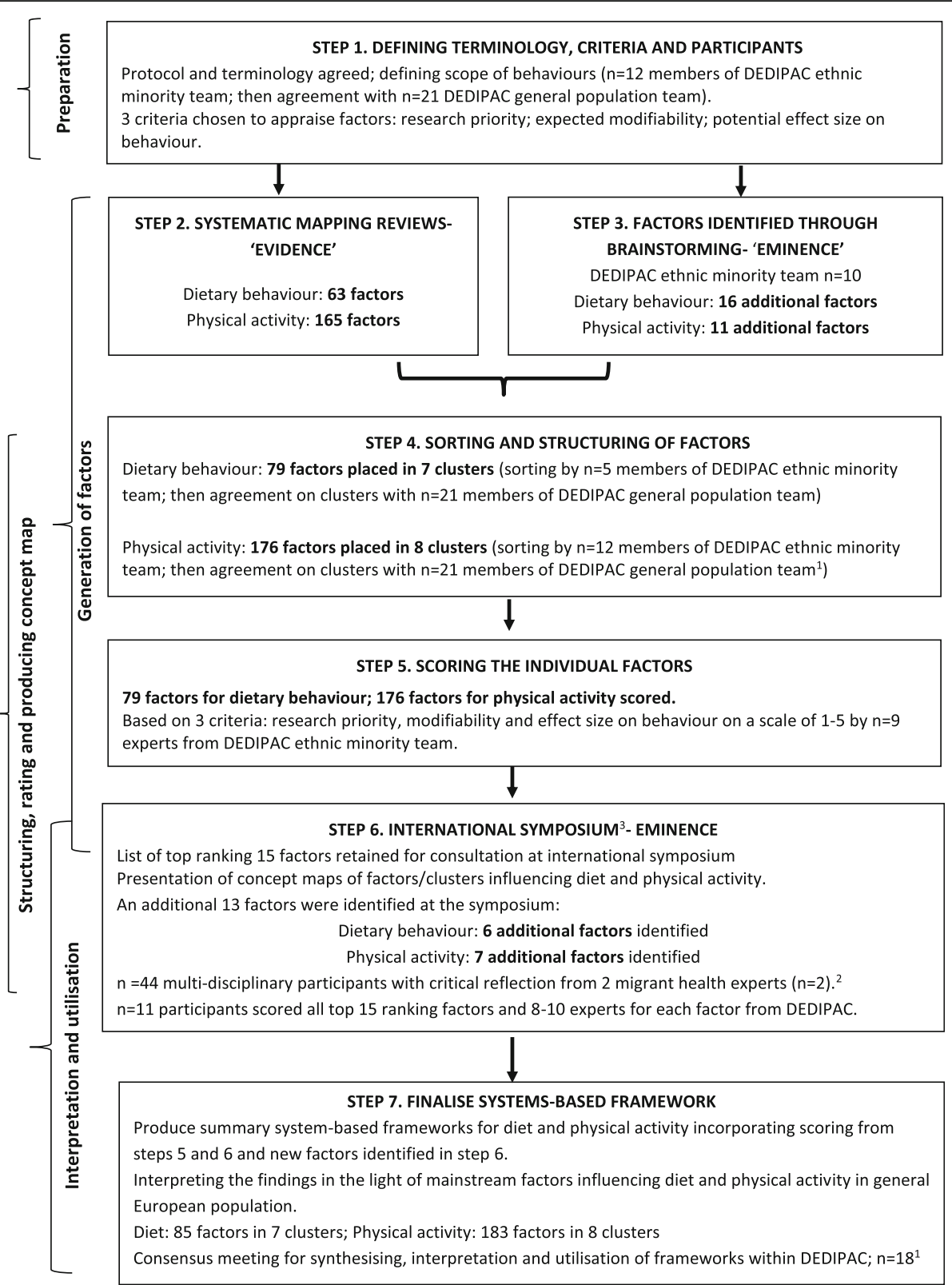

Fig. $1{ }^{1}$ Areas of expertise that were represented in the process are summarised in Table 1. ${ }^{2}$ Holdsworth M, Nicolaou M, Araba Saeed H, Jørun Langøien L, Powell K, Terragni L et al. (2015) Developing a framework map of the major determinants of dietary behaviour and physical activity/ sedentary behaviour in minority ethnic groups living in Europe. International Society for Behavioral Nutrition and Physical Activity, June, Edinburgh (UK), abstract book p107, S6.73. https://eventmobi.com/api/events/7231/documents/download/596c6adb-efOf-4695-be2c8a1927877d2c.pdf/as/ISBNPA\%202015\%20bstract\%20Book.pdf. Concept mapping process for developing the systems-based framework for dietary and physical activity behaviours in ethnic minority populations

all participants have to be involved in every step [32], so it was agreed that a small group would generate the factors and a larger group would sort and rate them.

\section{Generation of factors}

The next phase (Fig. 1- steps 2 and 3; then steps 4 and 6) involved generating a set of factors to represent the entire conceptual domain of the topic area, i.e. the factors that influence diet and physical activity (incorporating sedentary behaviours) of ethnic minority populations living in Europe. This was conducted in parallel for diet and physical activity behaviours from published 'evidence' and expert opinion ('eminence'). The aim was to develop wording that was detailed enough to convey the underlying meaning for each factor without requiring further explanation. 


\section{Published evidence (systematic mapping reviews)}

Factors were generated from published 'evidence' by conducting systematic mapping reviews (Fig. 1- step 2) of the factors influencing dietary behaviours [36] and physical activity behaviours (incorporating sedentary behaviours) [37] among minority groups living in Europe. The review methods and protocols were published elsewhere [PROSPERO database \#CRD42014013549/ \#CRD42014014575], but essentially primary data from quantitative and qualitative studies published over the 15 year period preceding data searching (1999-2014) were extracted. In synthesizing the findings, all of the factors (63 factors for diet; 165 factors for physical activity) were listed [36, 37]. Physical activity and sedentary behaviours were integrated together, as there was a lack of published research on sedentary behaviours of ethnic minority populations.

\section{Expert opinion ('eminence')}

Expert opinion was sought from three sources: brainstorming within the DEDIPAC ethnic minority team (Fig. 1- step 3) and by members of the DEDIPAC general population team (Fig. 1- step 4); and later by consultation with external experts during an international symposium (Fig. 1- step 6). Participants $(n=89$ in total throughout the process) were from a range of disciplines (Table 1), but they were not all involved at every step.

Factors that had not emerged from the reviews, but could be important, were generated by the DEDIPAC ethnic minority team (Fig. 1- step 3). Existing frameworks were also used to extract additional factors [13, 21, 23, 38]. An additional 40 factors (to those from the systematic mapping reviews) were identified at steps 3 and 6 combined ( 22 for diet and 18 for physical activity).

\section{Structuring, rating and producing a concept map Sorting and structuring of factors}

The emerging factors from the systematic mapping reviews and expertise within the DEDIPAC-KH were grouped into clusters, according to how they were seen to relate to each other. This resulted in 7 dietary behaviour clusters containing 79 factors and 8 physical activity clusters containing 176 factors (Additional file 1: Tables S1 and S2). This process was undertaken in two ways (Fig. 1- step 4). Firstly, during meetings of the DEDIPAC ethnic minority team, when the relationships between factors were collectively debated. Secondly, during a confirmatory stage involving members of the DEDIPAC ethnic minority and the general population teams. The concept map that emerged was discussed collectively, which led to some changes in wording of the clusters to enhance clarity and some factors were moved into different clusters (Fig. 1- step 4).

\section{Scoring the individual factors}

All of the factors were scored individually (Fig. 1- step 5) by DEDIPAC ethnic minority team members and some general population team members (Table 1) using the three criteria identified in the preparation phase, i.e. 'research priority', 'expected modifiability' and 'potential effect size on behaviour'. When scoring, individuals were asked to provide their scores based on their own professional judgments. The rating focus statement selected was: 'Score the following factors for their importance on a scale of 1 to 5 for dietary behaviour [or physical activity and sedentary behaviour] in ethnic minority groups', where $1=$ the lowest importance and $5=$ the highest. These three criteria were added together as a total measure of the perceived importance of individual factors for research and interventions. Individuals rated the factors separately and these scores were subsequently collated to develop the ranking of factors within clusters. The mean is the total sum divided by the number of factors, so a maximum of 15 could be gained for the three criteria on a Likert scale of $1-5$, with 5 as most modifiable, strongest effect, largest priority. The position that the factors were ranked in was based on the overall sum divided by the number of factors in each cluster (Table 4).

\section{International symposium- eminence}

Consultation took place during a dedicated symposium at an international conference (Fig. 1- step 6) [39]. Symposium participants scored the top 15 factors that had emerged from step 5 (Fig. 1) using the same three criteria. The decision to only request scoring of the top 15 factors was for pragmatic reasons as the symposium was time limited. The symposium also allowed reflection on the next phase of 'Interpretation and utilisation' of the framework.

\section{Interpretation and utilisation International symposium- eminence}

Two invited experts (external to DEDIPAC) in migrant health gave their views about the draft concept map, particularly where there were gaps in published literature and key research challenges for the future. This was followed by a short interactive discussion drawing on experiences and views from the audience $(n=44)$, during which, ideas were captured from symposium participants. The discussion resulted in the identification of a further 13 factors ( 6 for diet, 7 for physical activity), which were subsequently incorporated into the framework. This process led to a summary of the research challenges and knowledge gaps identified (Fig. 1- step 6).

\section{Finalise systems-based framework}

The rating of individual factors and clusters were assembled for the separate diet and physical activity frameworks. 
A face-to-face meeting of the DEDIPAC ethnic minority and general population teams was held to discuss the integrated framework (Fig. 1- step 7) and consensus was sought at the cluster level. It was the final step in the framework development process to compare the scoring of factors and ranking of clusters across minority ethnic populations with the general European population and to discuss the implications for research and interventions of the factors and clusters in the final framework.

Frameworks in the general population of factors influencing physical activity [29] and sedentary behaviour [30] separately, as well as for dietary behaviour [28] were developed in parallel by the general population teams in the DEDIPAC-KH. While the factors for ethnic minority populations fed into these general population frameworks, they were developed in separate processes [28-30]. This allowed for a post-hoc comparison of clusters identified in the general population with those identified in the ethnic minority framework.

\section{Results \\ Emerging clusters for dietary and physical activity behaviours}

Seven distinct clusters (containing 85 factors) were identified for dietary behaviour of ethnic minority populations (Additional file 1: Table S1): 'migration context'; 'social and cultural environment'; 'food beliefs and perceptions'; 'accessibility of food'; 'the body'; 'psychosocial'; and 'social and material resources'. The highest number of factors were identified in the 'social and cultural environment' cluster ( 20 factors), followed by 'food beliefs and perceptions' (13 factors). The 'psychosocial' and 'accessibility of foods' clusters had an equal number of factors (12 factors each). Only five factors were identified for 'the body' cluster.

Eight distinct clusters (containing 183 factors) were identified for physical activity behaviours (Additional file 1: Table S2): health and health communication; political environment; social and cultural environment; psychosocial; institutional environment; physical environment and opportunity; social and material resources; and migration context. The highest number of factors were identified in the social and cultural environment cluster (53 factors), followed by the psychosocial cluster (38 factors). Whilst the lowest number was identified for the political environment cluster (3 factors).

\section{Priority ranking of factors for dietary behaviour}

One-third of the top rated 15 factors for diet were related to food accessibility (Table 2). These factors scored highly in all criteria; and three of these factors (food availability, food policy, food price) were in the top five overall; indeed food price scored highest for its likely impact on population behaviour.
The factors in the 'food beliefs and perceptions' cluster that scored highly related to children's food preferences and the social role of food and food beliefs; although the latter two did not score well for modifiability, as they were seen as difficult to change, presumably because of their socially engrained nature. The psychosocial cluster of factors that reached the top 15 were related to perceived barriers, psychosocial stress and subjective norms influencing dietary behaviours. Perceived barriers in particular scored well for modifiability, suggesting barriers could be targeted in subsequent interventions. Even though so many factors emerged in the social and cultural cluster (Additional file 1: Table S1), only two of these were ranked highly enough across all criteria to be included in the final list of 15 factors (social networks; level of acculturation).

Only 'nutrition knowledge' scored well amongst the 'material and social resources' cluster (Table 2); the other factors in this cluster scored low on modifiability and therefore were not seen as imperative to study, e.g. income. Only 'immigrant related policy' in the host country scored well amongst the 'migration context' cluster, but even then it did not score well as a research priority. Others in this cluster had low scores for modifiability, such as the political context in the host country. No factors in 'the body' cluster emerged in the top 15 .

\section{Priority ranking of factors for physical activity}

Almost one-third of the top rated 15 physical activity factors were related to the 'physical environment and opportunity' cluster (Table 3). Two factors related to provision of culturally sensitive and/or women only facilities (Table 3). Four psychosocial factors were ranked highly overall, scoring well for modifiability (knowledge of physical activity, lack of physical activity skills, expectations of physical activity, attitudes).

'Physical activity at school' was ranked first, performing well in terms of its likely impact on population health, due to the potential reach that school based interventions can have, suggesting schools could be a priority setting in subsequent interventions. Only 'area deprivation' scored highly amongst the 'material and social resources' cluster, as it was seen to have an important impact on behaviours, but it scored less well for modifiability. Other factors related to material and social resources, such as income, scored less well, as they were seen as hard to modify.

Only three factors ranked highly (Table 2) amongst the 53 factors that emerged in the 'Social and cultural environment' cluster (Additional file 1: Table S2). Two of these appeared closely inter-related (social influence, habitus), where habitus was seen as how individuals perceive and react to the social world around them. The third factor (parental attitudes) reflects the high ranking given to children's physical activity behaviours. Of the 12 
Table 2 Ranking of top 15 dietary factors (presented in table are mean (SD))

\begin{tabular}{|c|c|c|c|c|c|c|}
\hline $\operatorname{Rank}^{a}$ & Factor & Modifiability & Priority for research & Effect on Behaviour & SUM & Cluster name \\
\hline 1 & Food availability & $3.37(1.18)$ & $3.50(1.00)$ & $3.93(1.10)$ & 10.80 & Accessibility of food \\
\hline 2 & Food policy & $3.27(0.96)$ & $3.95(0.94)$ & $3.57(1.19)$ & 10.78 & Accessibility of food \\
\hline 3 & Perceived barriers & $3.82(0.75)$ & $3.43(1.27)$ & $3.52(0.99)$ & 10.77 & Psychosocial \\
\hline 4 & Nutrition knowledge & $4.23(0.73)$ & $3.20(1.20)$ & $3.22(0.89)$ & 10.65 & Resources/social capital \\
\hline 5 & Food prices & $2.68(1.33)$ & $3.72(1.17)$ & $4.20(0.77)$ & 10.60 & Accessibility of food \\
\hline 6 & Children's food preferences & $3.43(0.75)$ & $3.35(0.93)$ & $3.72(0.91)$ & 10.50 & Food beliefs \& perceptions \\
\hline 7 & Food-related life-style & $2.95(1.00)$ & $3.70(0.98)$ & $3.77(1.11)$ & 10.42 & Accessibility of food \\
\hline 8 & Social role of food & $2.23(0.88)$ & $3.85(0.81)$ & $3.98(0.80)$ & 10.07 & Food beliefs \& perceptions \\
\hline 9 & Psycho-social stress & $2.95(1.00)$ & $3.65(1.09)$ & $3.45(0.94)$ & 10.05 & Psychosocial \\
\hline 10 & Food beliefs & $2.93(0.81)$ & $3.42(0.82)$ & $3.68(0.92)$ & 10.03 & Food beliefs \& perceptions \\
\hline 11 & Social networks & $2.55(0.83)$ & $3.62(0.59)$ & $3.68(0.73)$ & 9.85 & Social and cultural \\
\hline 12 & Subjective norms & $2.87(1.05)$ & $3.33(0.87)$ & $3.52(0.94)$ & 9.72 & Psychosocial \\
\hline 13 & Accessibility of traditional foods & $3.32(1.08)$ & $3.08(1.06)$ & $3.05(1.19)$ & 9.45 & Accessibility of food \\
\hline 14 & Immigrant related policy & $3.05(1.05)$ & $3.52(1.14)$ & $2.85(1.18)$ & 9.42 & Migration context \\
\hline 15 & Level of acculturation & $2.62(1.20)$ & $3.32(0.98)$ & $3.28(0.91)$ & 9.22 & Social and cultural \\
\hline
\end{tabular}

All scores on a scale of $1-5$, with 5 as most modifiable, strongest effect, largest priority

a Position that the factors were ranked in from the 79 diet factors based on 3 criteria of 'research priority', 'expected modifiability' and 'potential effect size on behaviour'; factors were scored by 20 people for all 3 criteria

factors in the 'health and health communication' cluster, only 'primary health care' was ranked highly. The high rating of primary health care in part stems from the central role of health professionals conveying the importance of physical activity, as it was seen as modifiable, with potential to reach many people through interventions. Only 'lack of knowledge of host culture' scored well in 'migration status', as it was seen as modifiable, whereas the other factors in this cluster were perceived as difficult to change. No factors in 'the political environment' emerged amongst those in the top 15.

\section{Cluster ranking for factors influencing dietary and physical activity behaviours}

For both diet and physical activity, 'psychosocial' factors was the top ranking cluster, which scored highly on all

Table 3 Ranking of top 15 factors related to physical activity behaviours (presented in table are mean (SD))

\begin{tabular}{|c|c|c|c|c|c|c|}
\hline Rank $^{a}$ & Factor & Modifiability & Priority for research & Effect on Behaviour & SUM & Cluster name \\
\hline 1 & Lack of physical activity at school & $3.82(1.10)$ & $3.53(0.86)$ & $3.67(0.77)$ & 11.02 & Institutional environment \\
\hline 2 & Knowledge of physical activity & $4.11(0.96)$ & $3.08(1.37)$ & $3.15(1.10)$ & 10.35 & Psychosocial \\
\hline 3 & Social influence & $2.86(1.02)$ & $3.60(1.09)$ & $3.78(0.88)$ & 10.25 & Social and cultural environment \\
\hline 4 & Lack of physical activity skills & $3.61(1.04)$ & $3.14(0.86)$ & $3.39(0.98)$ & 10.14 & Psychosocial \\
\hline 5 & Parental attitudes & $3.10(0.68)$ & $3.38(1.09)$ & $3.61(0.92)$ & 10.08 & Social and cultural environment \\
\hline 6 & Lack of culturally sensitive facilities & $3.28(1.07)$ & $3.44(0.92)$ & $3.33(0.84)$ & 10.05 & Physical environment and opportunity \\
\hline 7 & Expectations of physical activity & $3.33(1.08)$ & $3.22(1.06)$ & $3.45(1.10)$ & 10.00 & Psychosocial \\
\hline 8 & Attitudes & $3.38(0.98)$ & $3.27(1.07)$ & $3.35(1.03)$ & 10.00 & Psychosocial \\
\hline 9 & Facilities available & $3.05(1.06)$ & $3.38(0.78)$ & $3.50(0.96)$ & 9.94 & Physical environment and opportunity \\
\hline 10 & Access to a play area & $3.23(0.94)$ & $3.26(0.89)$ & $3.44(0.78)$ & 9.93 & Physical environment and opportunity \\
\hline 11 & Lack of knowledge of host culture & $3.72(0.96)$ & $2.94(0.87)$ & $3.12(0.68)$ & 9.78 & Migration context \\
\hline 12 & Area deprivation & $2.50(0.86)$ & $3.50(0.92)$ & $3.72(0.83)$ & 9.71 & Social and material resources \\
\hline 13 & Lack of women only facilities & $3.25(1.00)$ & $3.20(1.11)$ & $3.22(1.00)$ & 9.66 & Physical environment and opportunity \\
\hline 14 & Primary health care & $3.36(1.14)$ & $3.23(1.11)$ & $3.05(1.00)$ & 9.64 & Health and health communication \\
\hline 15 & Habitus & $2.60(1.14)$ & $3.23(1.40)$ & $3.63(1.29)$ & 9.46 & Social and cultural environment \\
\hline
\end{tabular}

All scores on a scale of $1-5$, with 5 as most modifiable, strongest effect, largest priority

a Position that the factors were ranked in from the 183 Physical activity/Sedentary behaviour factors based on the overall scores of the 3 criteria of 'research priority', 'expected modifiability' and 'potential effect size on behaviour'; factors were scored by 20 people for all 3 criteria 
three criteria, but particularly as a priority for research and its likely impact on behaviour (Table 4). The scores for the remaining clusters were ranked closely behind. The 'migration context' cluster of factors scored lowest, mainly because it had a low score for modifiability. Social and material resources were seen to have an important impact on behaviour, but scored less for modifiability. Overall, the relatively close ranking of the clusters for both behaviours suggested that all could have a part to play in developing interventions and research.

\section{A systems-based framework - A tool for prioritising research and interventions}

Figures 2 and 3 summarise the clusters that emerged and their priority ranking. The top scoring five factors in each cluster are highlighted.

An integrated framework for the major clusters of factors influencing both dietary and physical activity behaviours and the overlap between them is illustrated in the overall framework (Fig. 4).
The clusters of factors influencing the different behaviours were integrated to illustrate both similar and distinct clusters of factors. Four of the clusters were similar (psychosocial; social and cultural; social and material resources; and migration context) for diet and physical activity behaviours.

\section{Research priorities for ethnic minorities compared with those for the general host population}

Some similarities and differences were observed between ethnic minorities and general host populations. Similar sub-categories of clusters of factors emerged in the general population frameworks for diet [28], physical activity [29] and sedentary behaviours [30] combined, except for those in the migration context (Table 5).

The clusters of 'health and health communication' and 'institutional environment' did not emerge in the general population for physical activity; and 'material and social resources' did not emerge as a distinct cluster of factors influencing sedentary behaviours in the general population.

Table 4 Ranking of clusters for dietary and physical activity behaviour factors

\begin{tabular}{|c|c|c|c|c|c|c|c|}
\hline Cluster & $\begin{array}{l}\text { Number of } \\
\text { factors }^{\mathrm{a}}\end{array}$ & $\begin{array}{l}\text { Mean Modifiability } \\
\text { max score } 5\end{array}$ & $\begin{array}{l}\text { Mean Priority } \\
\text { for research } \\
\text { max score } 5\end{array}$ & $\begin{array}{l}\text { Mean effect on } \\
\text { behaviour } \\
\text { max score } 5\end{array}$ & $\begin{array}{l}\text { SUM of raw } \\
\text { scores }\end{array}$ & $\begin{array}{l}\text { Mean score/factor }{ }^{\mathrm{b}} \\
\text { max score } 15\end{array}$ & $\begin{array}{l}\text { Cluster } \\
\text { rank }^{c}\end{array}$ \\
\hline \multicolumn{8}{|l|}{ Dietary behaviour } \\
\hline Psychosocial & 12 & 3.07 & 3.10 & 3.69 & 108.43 & 9.04 & 1 \\
\hline $\begin{array}{l}\text { Food beliefs and } \\
\text { perceptions }\end{array}$ & 11 & 2.73 & 2.88 & 3.34 & 98.50 & 8.96 & 2 \\
\hline Social and material resources & 10 & 2.36 & 3.04 & 3.51 & 89.03 & 8.90 & 3 \\
\hline Accessibility of food & 12 & 2.46 & 3.02 & 3.31 & 105.57 & 8.80 & 4 \\
\hline The body & 5 & 2.87 & 2.54 & 3.14 & 42.75 & 8.55 & 5 \\
\hline $\begin{array}{l}\text { Social and cultural } \\
\text { environment }\end{array}$ & 18 & 1.99 & 2.84 & 3.19 & 144.27 & 8.02 & 6 \\
\hline Migration context & 13 & 1.47 & 2.51 & 3.08 & 91.70 & 7.05 & 7 \\
\hline \multicolumn{8}{|l|}{ Physical activity behaviours } \\
\hline Psychosocial & 38 & 2.70 & 2.64 & 3.17 & 323.62 & 8.52 & 1 \\
\hline $\begin{array}{l}\text { Institutional } \\
\text { environment }\end{array}$ & 14 & 2.68 & 2.29 & 3.00 & 114.73 & 8.20 & 2 \\
\hline Political environment & 3 & 2.14 & 2.95 & 3.05 & 24.44 & 8.15 & 3 \\
\hline $\begin{array}{l}\text { Social and cultural } \\
\text { environment }\end{array}$ & 49 & 2.13 & 2.64 & 3.12 & 394.32 & 8.05 & 4 \\
\hline $\begin{array}{l}\text { Physical environment } \\
\text { and opportunity }\end{array}$ & 31 & 2.45 & 2.47 & 3.08 & 247.81 & 7.99 & 5 \\
\hline $\begin{array}{l}\text { Social and material } \\
\text { resources }\end{array}$ & 12 & 1.83 & 2.66 & 3.41 & 94.71 & 7.89 & 6 \\
\hline $\begin{array}{l}\text { Health and health } \\
\text { communication }\end{array}$ & 12 & 2.29 & 2.35 & 3.22 & 94.34 & 7.86 & 7 \\
\hline Migration context & 17 & 1.76 & 2.31 & 2.70 & 121.90 & 7.17 & 8 \\
\hline
\end{tabular}

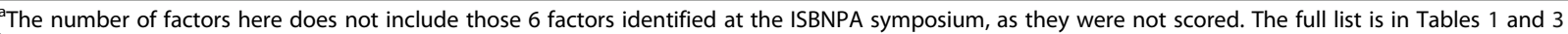
${ }^{b}$ Mean is the total sum divided by the number of factors- so a maximum of 15 could be gained as 3 criteria on a Likert scale of $1-5$, with 5 as most modifiable, strongest effect, largest priority

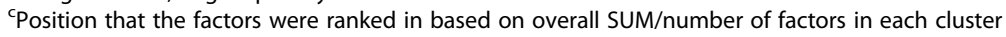




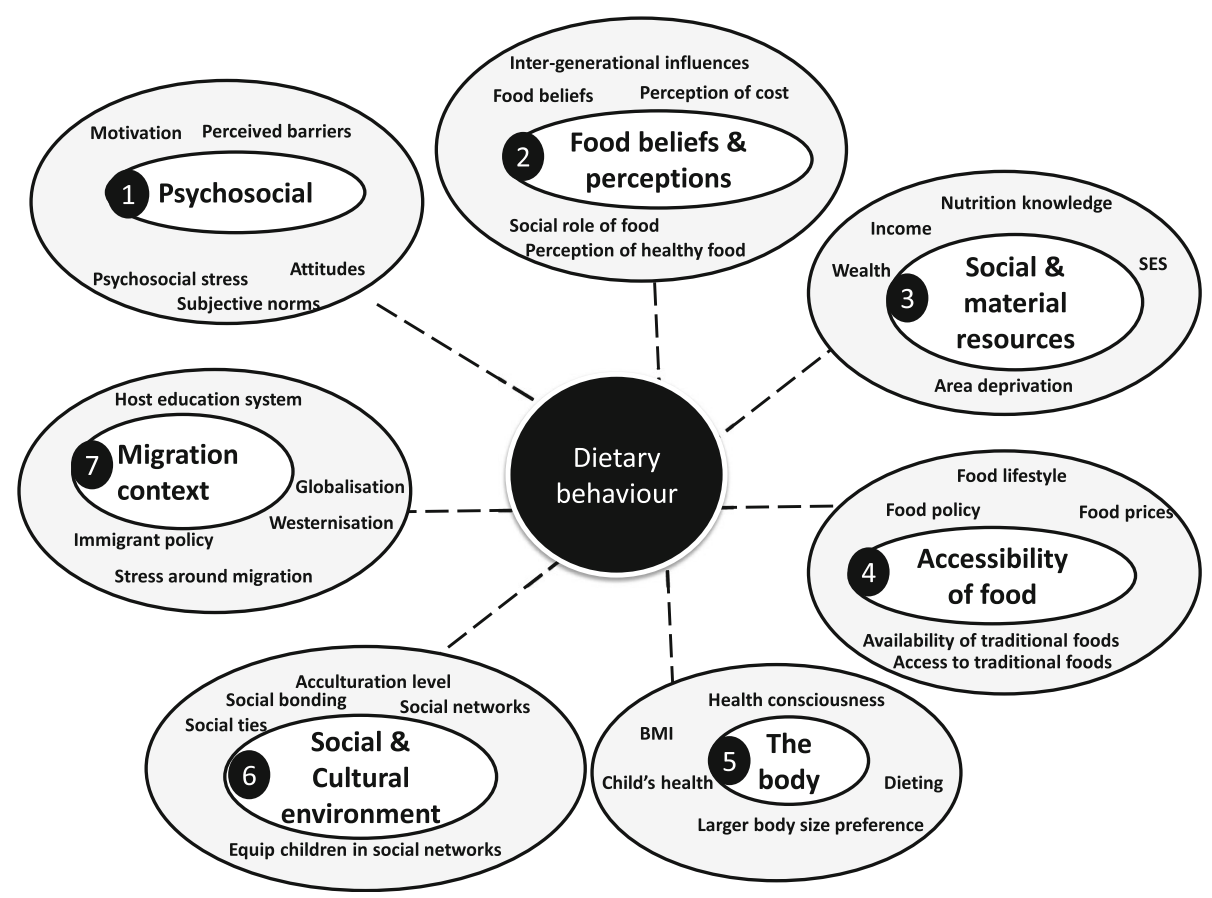

Fig. 2 A systems-based framework of the priority factors and clusters influencing dietary behaviours in ethnic minority populations living in Europe. The numbers represent the order of ranking of the clusters. The factors listed under each cluster are the top 5 factors ranked by a range of experts from a wide array of disciplines for their importance based on 3 criteria of 'research priority', 'expected modifiability' and 'potential effect size on behaviour'. Dotted lines indicate that factors in the cluster are associated with behaviours, but they do not indicate evidence for causation

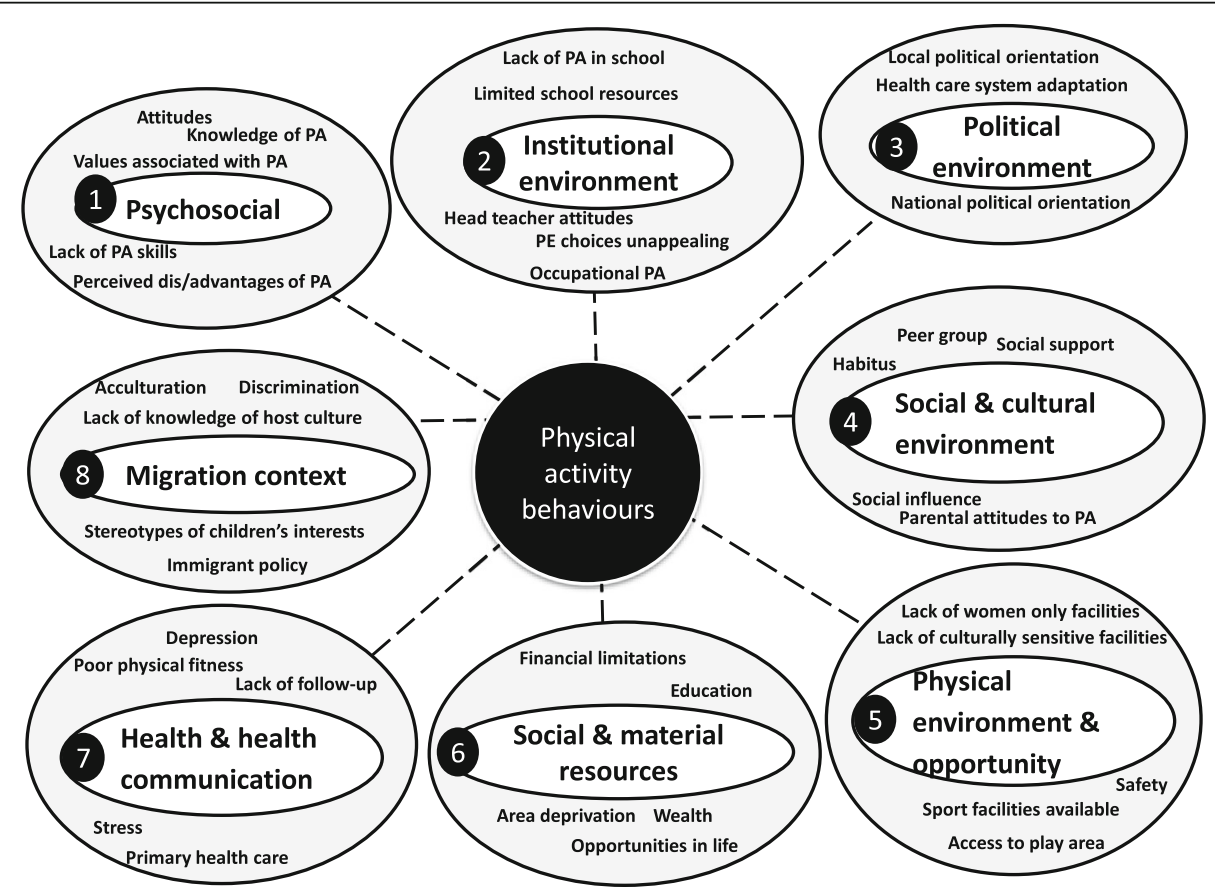

Fig. 3 A systems-based framework of the priority factors and clusters influencing physical activity behaviours in ethnic minority populations living in Europe. The numbers represent the order of ranking of the clusters. The factors listed under each cluster are the top 5 factors ranked by a range of experts from a wide array of disciplines for their importance based on 3 criteria of 'research priority', 'expected modifiability' and 'potential effect size on behaviour'. Dotted lines indicate that factors in the cluster are associated with behaviours, but they do not indicate evidence for causation 


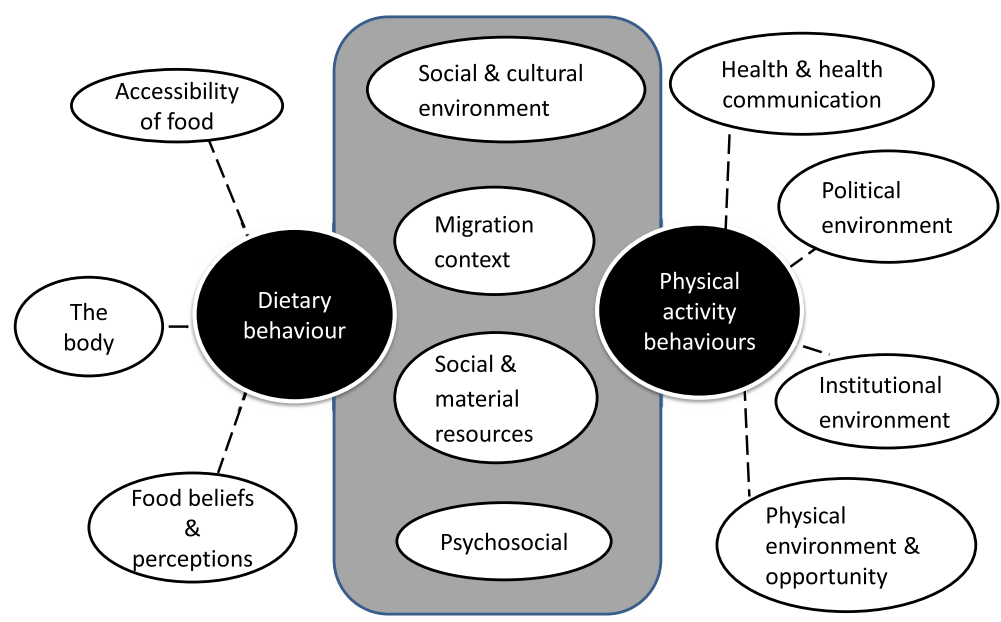

Fig. 4 A systems-based integrated framework of the clusters influencing dietary and physical activity behaviours in ethnic minority populations living in Europe. Dotted lines indicate that factors in the cluster are associated with behaviours, but they do not indicate evidence for causation

The 'social and cultural' cluster was ranked first for overall importance for its influence on physical activity amongst ethnic minority populations. Even though social and cultural factors emerged in the general population, the overall cluster was ranked lower for physical activity and sedentary behaviour (Table 5). The importance of psychosocial factors in the general population was ranked slightly less than for ethnic minority groups, as they were ranked in second and third position for sedentary behaviour and physical activity respectively. The political environment was ranked higher for its importance in influencing physical activity in ethnic minority populations than in the general population, where it was ranked last (sedentary behaviour) or next to last (physical activity) (Table 5).

For diet, the importance of 'psychosocial' factors, 'food beliefs and perceptions', 'social and material resources', and the 'social and cultural environment' were all ranked

Table 5 Comparing emerging clusters and their overall ranking in ethnic minorities with sub-categories in the general European population for dietary and physical activity behaviours

\begin{tabular}{|c|c|c|c|c|c|c|}
\hline \multicolumn{3}{|l|}{ Dietary behaviours } & \multicolumn{4}{|l|}{ Physical activity behaviours } \\
\hline \multirow[b]{2}{*}{ Cluster name } & \multicolumn{2}{|c|}{ Cluster Rank } & \multirow[b]{2}{*}{ Cluster name } & \multicolumn{3}{|c|}{ Cluster Rank } \\
\hline & $\begin{array}{l}\text { Ethnic } \\
\text { minorities }\end{array}$ & $\begin{array}{l}\text { General } \\
\text { population }^{a}\end{array}$ & & $\begin{array}{l}\text { Ethnic } \\
\text { minorities } \\
\text { (PA/SB) }\end{array}$ & $\begin{array}{l}\text { General population } \\
(\mathrm{PA})^{\mathrm{b}}\end{array}$ & $\begin{array}{l}\text { General population } \\
(\mathrm{SB})^{c}\end{array}$ \\
\hline Psychosocial & 1 & 7 & Psychosocial & 1 & 3 & 2 \\
\hline $\begin{array}{l}\text { Food beliefs and } \\
\text { perceptions }\end{array}$ & 2 & 17 & Institutional environment & 2 & - & 1 \\
\hline $\begin{array}{l}\text { Social and material } \\
\text { resources }\end{array}$ & 3 & 18 & Political environment & 3 & 5 & 6 \\
\hline $\begin{array}{l}\text { Accessibility of } \\
\text { food }\end{array}$ & 4 & 2 & $\begin{array}{l}\text { Social and cultural } \\
\text { environment }\end{array}$ & 4 & 2 and 4 & 5 \\
\hline The body & 5 & 30 & $\begin{array}{l}\text { Physical environment and } \\
\text { opportunity }\end{array}$ & 5 & 1 & 3 \\
\hline $\begin{array}{l}\text { Social and cultural } \\
\text { environment }\end{array}$ & 6 & 20 & Social and material resources & 6 & 6 & - \\
\hline \multirow[t]{2}{*}{ Migration context } & 7 & - & $\begin{array}{l}\text { Health and health } \\
\text { communication }\end{array}$ & 7 & - & 4 \\
\hline & & & Migration context & 8 & - & - \\
\hline
\end{tabular}

Matches here are based on overlap of the individual factors included in each sub-category ${ }^{\text {a }}$ or cluster but it should be noted that overlap may only be partial. All scores were ranked based on criteria including priority for research, modifiability and population-level effect (and relationship strength ${ }^{\mathrm{a}}$ )

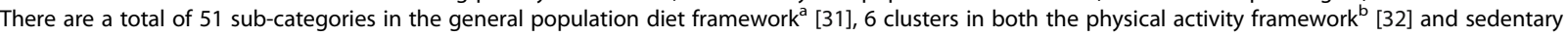
behaviour framework ${ }^{\mathrm{C}}[33]$

[PA = physical activity and SB = sedentary behaviour $]$ 
lower in the general population than for ethnic minority groups (Table 5). However, the importance of the cluster of factors relating to the 'accessibility of food' emerged as important across all populations.

\section{Discussion}

A system-based framework was established in this study to summarise the factors influencing dietary and physical activity behaviours in ethnic minority populations living in Europe. This is the first framework developed using a formal consensus methodology, drawing upon wide transdisciplinary evidence and eminence. It is envisaged that the framework will primarily be used as a tool to stimulate operationalisation and contextualisation for research and interventions.

There was insufficient evidence from specific ethnic minority groups, so therefore they were treated together, as there are shared experiences in the lives of people from ethnic minority populations that justify grouping them together. However, as with majority host populations, it is important to acknowledge the heterogeneity of ethnic minority populations living in Europe [36]. The different clusters are likely to interact, implying that factors in a specific cluster operate differently, depending on the factors in other clusters. In addition, clusters are highly dynamic, and might change over time as populations evolve, as the needs of ethnic minority populations are not static.

\section{Implications for research priorities for ethnic minority populations}

The framework highlights key research priorities for ethnic minority populations. For instance, addressing the highest rated factors associated with dietary behaviour would involve consideration of several clusters including 'accessibility of food,' 'psychosocial' and 'resources/social capital'. The clustering of factors in this way might precipitate a shift in the way complex behaviours are viewed; from a simple approach focusing on individual level factors, to a more holistic systems approach. In our study, psychosocial factors were ranked highly overall, particularly as they were seen as modifiable, which probably explains why research and interventions tend to focus on individual psychosocial factors [26].

The lack of evidence to attribute causation and effect strength is a major gap and research on causal models and pathways needs developing. Identifying and visualising inter-connections between factors remains difficult to do without data on their relationships, which requires more research taking a systems approach [22, 24, 26]. Both qualitative and longitudinal quantitative research provide useful insights for these inter-linkages and pathways that lead to dietary and physical activity behaviours $[36,40]$.
Practical considerations include the need for multidisciplinary researchers involved in understanding and changing behaviours to develop skills to evaluate the impact of complex, upstream, population-level interventions on the underlying clusters of factors [26], as well developing skills in cultural adaptation. Furthermore, in light of the peak migration in 2015-16 to Europe [41], new research regarding the impact of dietary behaviours on health for these populations is required, particularly amongst vulnerable migrant populations, including refugees, unaccompanied children and illegal migrants.

Research on ethnic minority populations often look through a lens of difference [36], i.e. focussing on what is different rather than similar with the general population, which may explain the wealth of social and cultural factors identified through this process. Although important, there is also scope for investigating commonalities, for example, how factors that drive dietary and physical activity behaviours in the majority population influence these behaviours amongst ethnic minority populations, which emphasises the needs for a systems approach across all populations. For example, one-third of the top rated 15 factors for diet were related to food accessibility, including food availability, how food policy shapes access to food and the price of food, which all emerged as important areas for research for the general population too. Interventions targeting these factors will require a whole population approach.

Most of the factors specific to the 'migration context' were not seen as a research priority, possibly because they would require studies involving several countries to research populations in different countries, and the context was seen as hard to change. The converse is also true, as research in the majority population seldom sheds light on how social and cultural factors influence behaviours, which may explain why they were ranked lower in the general population framework. The importance of research on collective behaviours, especially on the social practices that shape social habits and therefore practices around diet and physical activity is key for all population groups $[40,42]$, regardless of their ethnicity.

\section{Implications for developing interventions for ethnic minority populations}

The study's findings have highlighted that there are unlikely to be quick fixes or tipping points that can be isolated to change behaviours, rather, several factors could be targeted to improve diet and/or physical activity behaviours across the inter-related system of clusters. This reinforces the need for a systems approach in planning multifaceted interventions in order to account for the (sometimes unexpected) interaction between the factors influencing behaviour. Conventional approaches focusing on individual level behaviour change are insufficient [26]. 
The contextualisation for interventions is crucial too, which is in contrast with the high priority given to the individual level psychosocial factors in this study. This finding is largely a reflection of prevailing perceptions that these are easy to change. However, changing individual level factors, whilst the context remains the same, is insufficient to drive behaviour change, in view of the socially and culturally embedded nature of dietary [43] and physical activity behaviours.

This study's findings have highlighted much commonality between the factors influencing the behaviours in ethnic minority groups and the majority population. This begs the question of whether interventions are needed that address factors that are specific to ethnic minority groups, or whether mainstream interventions should be encouraged that can reach all groups. The study suggests that 'mainstream' interventions targeting the general population could address many factors identified as there was much in common between minority and host populations, such as food policy, food pricing, physical activity at school, access to play areas, area-level deprivation and so forth. However, even if factors are shared, their importance and focus might differ, e.g. the need for women only facilities, the social role of food might be stronger in more collective cultures. There are specific factors in the context of migration that will need to be addressed at a higher policy level, including policies encouraging integration.

Two different approaches for developing interventions that can reach ethnic minority populations are advocated for [44]. These consist of either adapting mainstream interventions for the majority population to be 'diversity sensitive', so that they can be equally effective for all citizens regardless of their cultural, religious or ethnic background, or alternatively developing 'migrant-specific' interventions by culturally adapting services and interventions to individual backgrounds of specific minority ethnic groups. The framework developed could be used to develop either approach, as well as encourage new approaches. Most of the evaluations of culturally sensitive interventions have been conducted in the US $[45,46]$ and may not be transferable. Additionally, evidence has indicated that evaluations lack explicit information about the components of cultural adaptation, and little or no detail is provided regarding how interventions are cultural adapted [47]. Evaluating interventions in a way that goes beyond 'what works', but also identifies 'for whom it works and in what context', such as realist approaches [48] would be well adapted to unravel the underlying processes. It should also be emphasised that this framework does not provide ready-made answers for intervention development. As for the case for 'majority' populations, a needs assessment will remain a necessary part of the process [49].

\section{Methodological limitations}

An important limitation was that whilst the frameworks for physical activity and sedentary behaviour followed a very similar concept mapping approach to determining clusters of factors, the framework for dietary behaviours did not include emerging clusters [28]. The dietary behaviour framework was developed by sorting individual factors into pre-defined categories with a positivist topdown process, using a socio-ecological approach [31]. Whereas a constructivist approach was taken in the development of the other frameworks, as clusters emerged from the data. However, both approaches are holistic, given that the clusters that emerged were so similar with the different approaches. In addition, factors were rated in a different manner in the general population's dietary behaviour framework as the overall priority for research was based on a weighted average of ratings for their modifiability, relationship strength and population-level effect [28].

Another limitation is that research participants did not cluster factors as individuals, but collectively as a group, meaning that clusters could not be created mathematically using cluster analysis [32]. This approach was decided against due to time limitations during data collection for individuals to cluster factors together separately. Even in the case of individual clustering, the results of cluster analysis often require visible adjustment to make them meaningful [32].

The decision to only request scoring of the top 15 ranking factors in the international symposium may have introduced bias, as it is unknown if these factors would be different if other people from within the broader DEDIPAC-KH or from outside it had participated in the rating of all factors. However, new/additional factors were explicitly sought from external participants, in order to compensate for this limitation.

The concept mapping exercise led to a hypothesised ranking of factors within clusters, while the systems approach implies that the interaction of context and interrelated factors is what influences behaviour [26]. However, evidence for links between factors was not found, due to a lack of research on the underlying mechanisms. Given this, there remains uncertainty about the specific factors that are a priority for research. A further limitation was the profile of participants. The framework was informed by academic researchers and although some have extensive experience with ethnic minority and migrant origin populations, input from individuals from agencies who work closely with different populations would have improved its completeness. Academic specialists in migrant health were invited as experts, enhancing confidence in the potential utility of the framework presented.

Other limitations included the lack of research highlighting the drivers of dietary and physical activity 
behaviours across the life-course, as most research targeted adults. There were insufficient studies to differentiate by age or life-course and further research is particularly required on children and adolescents, as they clearly have a role in influencing behaviours of the whole family. There was limited research on sedentary behaviour among ethnic minorities. Some ethnic minority groups were under-represented, particularly recent migrant populations to Europe, which should also be a priority for research.

\section{Conclusions}

This is the first systems-based framework to be developed that sheds light on dietary and physical activity behaviours of ethnic minority populations living in Europe, drawing on both evidence and eminence. Distinct clusters emerged for both dietary and physical activity behaviours, of which four clusters were similar across behaviours (social and cultural environment; social and material resources; psychosocial; and migration context), suggesting that an integrated approach for interventions across behaviours in these clusters of factors could bring maximum benefit. Similar clusters of factors emerged in the majority population frameworks for diet and physical activity behaviours, but their relative importance differed for ethnic minority populations, compared with the majority population.

Our findings illustrate that innovative research and interventions need to be developed that are sensitive to the needs of ethnic minority populations. Dietary and physical activity behaviours are intransigent and addressing them will require enormous innovation. A systems approach may help in shifting the current public health paradigm towards a more holistic approach that considers what works for whom and in what context, in order to ensure that ethnic minorities are included, alongside mainstream European populations.

\section{Additional file}

Additional file 1: Table S1. Concept map of the 85 factors and 7 clusters that emerged influencing dietary behaviours in ethnic minority groups. Table S2. Concept map of the 183 factors and the 8 clusters that emerged influencing physical activity behaviours (DOCX $46 \mathrm{~kb}$ )

\section{Abbreviations}

BMI: Body mass index; DEDIPAC: DEterminants of Dlet and Physical ACtivity; PA: Physical activity; SB: Sedentary behaviour; SES: Socioeconomic status

\section{Acknowledgements}

Not applicable.

\section{Funding}

The preparation of this paper was supported by the Determinants of Diet and Physical ACtivity (DEDIPAC) knowledge hub. This work is supported by the Joint Programming Initiative 'Healthy Diet for a Healthy Life'. The funding agencies supporting this work are (in alphabetical order of participating
Member State in this paper): Belgium: Research Foundation - Flanders; France: Institut National de la Recherche Agronomique (INRA); Germany: Federal Ministry of Education and Research; Italy: Ministero dell'Istruzione, Università e Ricerca, Italy; Norway: The Research Council of Norway; The Netherlands: The Netherlands Organisation for Health Research and Development (ZonMw); The UK: The Medical Research Council (MRC). The authors would like to thank the entire thematic area 2 of the DEDIPAC project.

Availability of data and materials

All data generated or analysed during this study are included in this published article and its Additional file.

\section{Authors' contributions}

All authors conceptualized and designed the study. $\mathrm{MH}$ drafted the manuscript. All authors reviewed draft versions of the manuscript and provided suggestions and critical feedback. All authors have made a significant contribution to this manuscript and approved the final manuscript.

\section{Ethics approval and consent to participate}

This study involved experts in virtue of their training and expertise during an international symposium. Experts have information and knowledge in a substantive area beyond that of the average person and they regularly share this information and knowledge through consultation, teaching or public speaking, or publications and written reports. The Medical Ethics Review Committee of the Academic Medical Center (Netherlands) stated that ethical approval was not required (reference number W16_388 \# 16.454) because the Medical Research Involving Human Subjects Act (WMO) did not apply to our study, as expert participants are not classified as human subjects when asked to provide opinions within their areas of expertise. No personal information was collected about the experts, and only their expert opinion about the factors influencing dietary and physical activity behaviours was collected in a confidential way.

Consent for publication

Not applicable.

\section{Competing interests}

The authors declare that they have no competing interests.

\section{Publisher's Note}

Springer Nature remains neutral with regard to jurisdictional claims in published maps and institutional affiliations.

\section{Author details}

${ }^{1}$ Public Health Section, School of Health and Related Research-ScHARR, The University of Sheffield, Sheffield, UK. ${ }^{2}$ Academic Medical Centre, University of Amsterdam, Department of Public Health, Amsterdam Public Health research Institute, Amsterdam, The Netherlands. ${ }^{3}$ Department of Physical Education, Norwegian School of Sport Sciences, Oslo, Norway. ${ }^{4}$ Institute for Applied Health Research, School of Health and Life Science, Glasgow Caledonian University, Glasgow, UK. ${ }^{5}$ Department of Psychological Assessment and Health Psychology, University of Konstanz, Constance, Germany.

${ }^{6}$ Department of Movement, Human and Health Sciences, University of Rome Foro Italico, Rome, Italy. 'Department of Nutrition, University of Oslo, Oslo, Norway. ${ }^{8}$ Department of Nursing and Health Promotion Faculty of Health Sciences, Oslo and Akershus University College of Applied Sciences, Oslo, Norway. ${ }^{~}$ UKCRC Centre for Diet and Activity Research, MRC Epidemiology Unit, University of Cambridge School of Clinical Medicine, Box 285, Institute of Metabolic Science, Cambridge Biomedical Campus, Cambridge CB2 OQQ, UK. ${ }^{10}$ Present address: Department of Nutrition and Exercise Physiology, Elson S Floyd College of Medicine, Washington State University, Spokane WA 99210-1495, USA. 11Department of Public Health, Ghent University, Ghent, Belgium. ${ }^{12}$ Department of Public Health, Ghent University, Ghent, Belgium. ${ }^{13}$ Consumption Research Norway SIFO, Oslo and Akershus University College of Applied Sciences, Oslo, Norway. ${ }^{14}$ UMR MOISA, Campus INRA-SupAgro de la Gaillarde, Montpellier, France. 
Received: 5 February 2017 Accepted: 24 October 2017 Published online: 07 November 2017

\section{References}

1. Faskunger J, Eriksson U, Johansson SE, Sundquist K, Sundquist J. Risk of obesity in immigrants compared with swedes in two deprived neighbourhoods. BMC Public Health. 2009;9(1):304.

2. Patel J, Vyas A, Cruickshank J, Prabhakaran D, Hughes E, Reddy K, et al. Impact of migration on coronary heart disease risk factors: comparison of Gujaratis in Britain and their contemporaries in villages of origin in India. Atherosclerosis. 2006;185(2):297-306.

3. Leung G, Stanner S. Diets of minority ethnic groups in the UK: influence on chronic disease risk and implications for prevention. Nutr Bull. 2011;36(2):161-98.

4. Hayes L, White M, Unwin N, Bhopal R, Fischbacher C, Harland J, et al. Patterns of physical activity and relationship with risk markers for cardiovascular disease and diabetes in Indian, Pakistani, Bangladeshi and European adults in a UK population. J Public Health. 2002;24(3):170-8.

5. Babakus WS, Thompson JL. Physical activity among south Asian women: a systematic, mixed-methods review. Int J Behav Nutr Phys Act. 2012;9(1):1.

6. Besharat Pour M, Bergström A, Bottai M, Kull I, Wickman M, Håkansson N, et al. Effect of parental migration background on childhood nutrition, physical activity, and body mass index. J Obes. 2014; https://doi.org/10.1155/2014/ 406529.

7. De Munter JS, Agyemang C, Van Valkengoed IG, Bhopal R, Zaninotto P, Nazroo J, et al. Cross national study of leisure-time physical activity in Dutch and English populations with ethnic group comparisons. Eur J Pub Health. 2013;23(3):440-6.

8. Hansen AR, Ekholm O, Kjøller M. Health behaviour among non-western immigrants with Danish citizenship. Scandinavian Journal of Public Health. 2008;36(2):205-10.

9. Hornby-Turner YC, Hampshire KR, Pollard TMA. Comparison of physical activity and sedentary behaviour in 9-11 year old British Pakistani and white British girls: a mixed methods study. Int J Behav Nutr Phys Act. 2014;11(1):1.

10. Dagkas S, Benn T. Young Muslim women's experiences of Islam and physical education in Greece and Britain: a comparative study. Sport Educ Soc. 2006;11(1):21-38.

11. Södergren M, Hylander I, Törnkvist L, Sundquist J, Sundquist K. Arranging appropriate activities: immigrant women's ideas of enabling exercise. Womens Health Issues. 2008;18(5):413-22.

12. Rawlins E, Baker G, Maynard M, Harding S. Perceptions of healthy eating and physical activity in an ethnically diverse sample of young children and their parents: the DEAL prevention of obesity study. J Hum Nutr Diet. 2013; 26(2):132-44.

13. Satia-About a J, Patterson RE, Neuhouser ML, Elder J. Dietary acculturation: applications to nutrition research and dietetics. J Am Diet Assoc. 2002; 102(8):1105-18,

14. Brustad M, Parr C, Melhus M, Lund E. Childhood diet in relation to Sami and Norwegian ethnicity in northern and mid-Norway-the SAMINOR study. Public Health Nutr. 2008;11(02):168-75.

15. Nicolaou M, Doak CM, van Dam RM, Brug J, Stronks K, Seidell JC. Cultural and social influences on food consumption in Dutch residents of Turkish and Moroccan origin: a qualitative study. J Nutr Educ Behav. 2009;41(4): 232-41.

16. Lawton J, Ahmad N, Hanna L, Douglas M, Bains H, Hallowell N. 'We should change ourselves, but we can't': accounts of food and eating practices amongst British Pakistanis and Indians with type 2 diabetes. Ethn Health. 2008;13(4):305-19.

17. Andersen E, Høstmark AT, Holme I, Anderssen SA. Intervention effects on physical activity and insulin levels in men of Pakistani origin living in Oslo: a randomised controlled trial. Immigr Minor Health. 2013;15(1):101-10.

18. Kay T. Daughters of Islam family influences on Muslim young Women's participation in sport. International Review for the Sociology of Sport. 2006; 41(3-4):357-73.

19. Méjean C, Traissac P, Eymard-Duvernay S, Delpeuch F, Maire B. Influence of acculturation among Tunisian migrants in France and their past/present exposure to the home country on diet and physical activity. Public Health Nutr. 2009;12(06):832-41

20. Pollard TM, Guell C. Assessing physical activity in Muslim women of south Asian origin. J Phys Act Health. 2012;9(7):970.

21. Kumanyika SK, Obarzanek E, Stettler N, Bell R, Field AE, Fortmann SP, et al. Population-based prevention of obesity: the need for comprehensive promotion of healthful eating, physical activity, and energy balance: a scientific statement from American Heart Association Council on epidemiology and prevention, interdisciplinary Committee for Prevention. Circulation. 2008:118(4):428-64.

22. Butland B, Jebb S, Kopelman P, McPherson K, Thomas S, Mardell J, et al. Foresight. Tackling obesities: future choices. Project report. Foresight. Tackling obesities: future choices. In: Project report; 2007. https://www.gov. uk/government/publications/reducing-obesity-future-choices.

23. Delavari M, Farrelly A, Renzaho A, Mellor D, Swinburn B. Experiences of migration and the determinants of obesity among recent Iranian immigrants in Victoria, Australia. Ethn Health. 2013;18(1):66-82.

24. Carey G, Malbon E, Carey N, Joyce A, Crammond B, Carey A. Systems science and systems thinking for public health: a systematic review of the field. BMJ Open. 2015;30(5):12.

25. Homer J, Hirsch G. Opportunities and demands in public health systems. Am J Public Health. 2006;96(3):452-8.

26. Rutter $\mathrm{H}$, Savona N, Glonti K, et al. The need for complex systems model of evidence for public health. Lancet. 2017; https://doi.org/10.1016/S01406736(17)31267-9.

27. Lakerveld J, Van Der Ploeg HP, Kroeze W, Ahrens W, Allais O, Andersen LF, et al. Towards the integration and development of a cross-European research network and infrastructure: the DEterminants of Dlet and physical ACtivity (DEDIPAC) knowledge hub. Int J Behav Nutr Phys Act 2014;11(1):1.

28. Stok FM, Hoffmann S, Volkert D, Boeing H, Ensenauer R, Stelmach-Mardas M, et al. The DONE framework: Creating, evaluating and updating of an interdisciplinary, dynamic framework 2.0 of determinants of nutrition and eating. PLoS One 2017;2;12(2):e0171077. doi:https://doi.org/10.1371/journal. pone. 0171077 .

29. Condello G, Ling FC, Bianco A, Chastin S, Cardon G, Ciarapica D, et al. Using concept mapping in the development of the EU-PAD framework (EUropean physical activity determinants across the life course): a DEDIPAC-study. BMC Public Health. 2016;16(1):1145.

30. Chastin S, De Craemer M, Lien N, Bernaards C, Buck C, Oppert J, et al. The SOS-framework (Systems of Sedentary behaviours): an international transdisciplinary consensus framework for the study of determinants, research priorities and policy on sedentary behaviour across the life course: a DEDIPAC-study. Int J Behav Nutr Phys Act. 2016;13(1):83. https://doi.org/ 10.1186/s12966-016-0409-3.

31. Booth SL, Sallis JF, Ritenbaugh C, Hill JO, Birch LL, Frank LD, et al. Environmental and societal factors affect food choice and physical activity: rationale, influences, and leverage points. Nutr Rev. 2001;59:S21-36.

32. Trochim WM. An introduction to concept mapping for planning and evaluation. Evaluation and program planning. 1989;12(1):1-16.

33. Daley BJ. Using concept maps in qualitative research. Theory, Methodology, Technology. Proc. of the First Int. Conference on Concept mapping Pamplona, Spain: Concept Maps; 2004.

34. Wheeldon J, Faubert J. Framing experience: concept maps, mind maps, and data collection in qualitative research. Int J Qual Methods. 2009;8:3.

35. Leischow SJ, Milstein B. Systems thinking and modeling for public health practice. Am J Public Health. 2006;96(3):403-5

36. Osei-Kwasi HA, Nicolaou M, Powell K, Terragni L, Maes L, Stronks K, et al. Systematic mapping review of the factors influencing dietary behaviour in ethnic minority groups living in Europe: a DEDIPAC study. Int J Behav Nutr Phys Act. 2016;13(1):1

37. Langøien $\sqcup$, Terragni L, Rugseth G, Nicolaou M, Holdsworth M, Stronks K, Lien N, Roos G. Systematic mapping review of the factors influencing physical activity and sedentary behaviour in ethnic minority groups in Europe: a DEDIPAC study. Int J Behav Nutr Phys Act. 2017;14:99.

38. Kockturk-Runefors TA. Model for adaptation to a new food pattern: the case of immigrants. Appetite. 1991;16(2):163.

39. Holdsworth M, Nicolaou M, Osei-Kwasi HA, Langøien L, Powell K, Terragni $L$, et al. Developing a framework map of the major determinants of dietary behaviour and physical activity/sedentary behaviour in minority ethnic groups living in Europe. Edinburgh (UK): abstract book of the conference of International Society for Behavioral Nutrition and Physical Activity (https:// eventmobi.com/api/events/7231/documents/download/596c6adb-efof4695-be2c-8a1927877d2c.pdf/as/ISBNPA\%202015\%20Abstract\%20Book.pdf); 2015. p107, S6.73

40. Powell K, Wilcox J, Clonan A, Bissell P, Preston L, Peacock M, et al. The role of social networks in the development of overweight and obesity among adults: a scoping review. BMC Public Health. 2015;15(1):1. 
41. International organisation for Migration. Migration flows to Europe- 2017 Quarterly overview. 2017. http://migration.iom.int/docs/Q1_2017_statistical_ Overview.pdf. Accessed 10 July 2017.

42. Shove E, Pantzar M, Watson M. The dynamics of social practice: everyday life and how it changes. London: Sage Publications; 2012.

43. Delormier T, Frohlich KL, Potvin L. Food and eating as social practiceunderstanding eating patterns as social phenomena and implications for public health. Sociol Health IIIn. 2009;31(2):215-28.

44. Razum O, Spallek J. Addressing health-related interventions to immigrants: migrant-specific or diversity-sensitive. Int J Public Health. 2014;59(6):893-5.

45. Fisher TL, Burnet DL, Huang ES, et al. Cultural leverage: interventions using culture to narrow racial disparities in health care. Med Care Res Rev. 2007; 64:243S-82S.

46. Neamah HH, Sebert Kuhlmann AK, Tabak RG. Effectiveness of program modification strategies of the diabetes prevention program: a systematic review. Diabetes Educ. 2016;42(2):153-65.

47. Diaz E, Ortiz Barreda G, Ben-Shlomo Y, et al. Interventions to improve immigrant health. A scoping review. Eur J Pub Health. 2017;27(3):433-9.

48. Harris J, Springett J, Booth A, et al. Can community-based peer support promote health literacy and reduce inequalities? A realist review. J Public Health Res. 2015;3(3)

49. Bartholomew LK, Parcel GS, Kok G. Intervention Mapping: A Process for Developing Theory and Evidence-Based Health Education Programs. 1998; 25(5):545-63.

Submit your next manuscript to BioMed Central and we will help you at every step:

- We accept pre-submission inquiries

- Our selector tool helps you to find the most relevant journal

- We provide round the clock customer support

- Convenient online submission

- Thorough peer review

- Inclusion in PubMed and all major indexing services

- Maximum visibility for your research

Submit your manuscript at www.biomedcentral.com/submit
Biomed Central 